\title{
Associations between energy density of meals and snacks and overall diet quality in British adults
}

\author{
K. Murakami ${ }^{1}$ and M.B.E. Livingstone ${ }^{2}$ \\ ${ }^{1}$ Department of Nutrition, School of Human Cultures, University of Shiga Prefecture, Shiga 522 8533, Japan and \\ ${ }^{2}$ Northern Ireland Centre for Food and Health, Ulster University, Coleraine BT52 1SA, UK
}

Understanding how the energy density (ED) of different combinations of foods in meals and snacks influence overall diet quality is important for, for example, the development of food-based guidelines. However, virtually no research has been conducted in this area, mainly because there is no consensus about what constitutes a snack, a meal, or an eating occasion ${ }^{(1)}$. The present cross-sectional study examined how the ED of meals and snacks influence overall diet quality.

The analytic sample included 1451 British adults (659 men and 792 women) aged 19-64 years in the National Diet and Nutrition Survey. Based on data from 7-d weighed dietary record, all eating occasions were divided into meals or snacks based on contribution to total energy intake (EI) (meals: $\geqslant 15 \%$; snacks: $<15 \%)^{(2)}$. ED (kJ/g) of meals and snacks was calculated based on solid foods only and excluding all caloric and non-caloric beverages. Overall diet quality was assessed using the Healthy Diet Indicator (HDI) ${ }^{(3)}$ and Mediterranean Diet Score (MDS) $)^{(4)}$.

While $\geqslant 75 \%$ of overall EI was derived from meal, ED of meals was lower than ED of snack in both men (mean (SD): 7.75 (1.33) v $9.57(4.31) \mathrm{kJ} / \mathrm{g} ; P<0.0001)$ and women (mean (SD): $7.19(1.45)$ v $8.10(4.26) \mathrm{kJ} / \mathrm{g} ; P<0.0001)$. After adjustment for age and social class, both ED of meals and ED of snacks were inversely associated with both HDI and MDS $(P \leqslant 0 \cdot 002)$, but stronger associations were observed for meals relative to snacks. One-unit increase of ED of meals and ED of snacks decreased HDI by 0.25 (SE: 0.04) and 0.07 (SE: 0.01) in men, respectively, and by 0.29 (SE: 0.03) and 0.04 (SE: 0.01) in women, respectively. The corresponding values for MDS were 0.36 (SE: 0.05) and 0.09 (SE: 0.01) (men) and 0.43 (SE: 0.04) and 0.06 (SE: 0.01) (women). Similar results were obtained when meals and snacks were defined based on time of consumption (meals: 06.00-10.00, 12.00-15.00 and 18.00-21.00 hours; snacks: all others) ${ }^{(5)}$ or when only acceptable reporters of EI (ratio of EI to estimated energy requirement $0 \cdot 665-1 \cdot 335^{(6)} ; n 868$ ) were analysed.

In conclusion, increased ED of meals and to a lesser extent, snacks, was associated with lower overall diet quality in British adults, irrespective of the definition of meals and snacks. These associations suggest that overall diet quality may be improved by focusing specifically on lowering the ED of meals.

This work was supported in part by the Grants-in-Aid for Young Scientists (B) from the Ministry of Education, Culture, Sports, Science and Technology of Japan (grant number 15K16213).

1. Leech RM, Worsley A, Timperio A, et al. (2015) Nutr Res Rev 28, 1-21.

2. Ritchie LD (2012) Am J Clin Nutr 95, 290-296.

3. Huijbregts P, Feskens E, Rasanen L, et al. (1997) BMJ 315, 13-17.

4. Trichopoulou A, Orfanos P, Norat T, et al. (2005) BMJ 330, 991.

5. Duffey KJ, Pereira RA \& Popkin BM (2013) Eur J Clin Nutr 67, 868-874.

6. Murakami K, McCaffrey TA \& Livingstone MBE (2013) Br J Nutr 110, 2047-2057. 